\section{Could cardiovascular disease risk stratification and management in rheumatoid arthritis be enhanced?}

\author{
Patrick H Dessein, ${ }^{1}$ Anne G Semb ${ }^{2}$
}

The markedly enhanced risk of atherosclerotic cardiovascular disease (CVD) in rheumatoid arthritis (RA) is well documented. ${ }^{1} 2$ This prompted a European League Against Rheumatism (EULAR) task force to make a commendable effort in producing recommendations for cardiovascular risk management in patients with inflammatory arthritis. ${ }^{3}$ These included the application of the systematic coronary risk evaluation score (SCORE), a multiple major traditional risk factor assessment equation. In addition, the EULAR task force recommended applying a multiplier of 1.5 in patients with RA that met 2 of 3 criteria consisting of (1) a disease duration $>10$ years, (2) rheumatoid factor or anticyclic citrullinated peptide positivity and (3) the presence of extra-articular manifestation, thereby creating the modified (m) SCORE.

Risk factor assessment algorithms, including the SCORE and the Framingham risk equation, are recommended worldwide as part of CVD risk management in the population at large. ${ }^{4}$ These equations allow for stratifying subjects into low, intermediate, high and very high risk groups. With regard to CVD risk management, lifestyle factors should be addressed in all individuals. The use of cardiovascular drugs, particularly antihypertensive and lipid-lowering agents should be considered in those at high or very high risk as these interventions markedly reduce CVD event rates in such persons. Patients with established CVD, diabetes and chronic kidney disease are at high or very high risk and hence, risk factor equation application is not indicated.

Nonetheless, approximately a third of CVD events are not attributable to major

\footnotetext{
${ }^{1}$ Cardiovascular Pathophysiology and Genomics Research Unit, School of Physiology, Faculty of Health Sciences, University of the Witwatersrand, Johannesburg, South Africa; ${ }^{2}$ Department of Rheumatology, Diakonhjemmet Hospital, Oslo, Norway

Correspondence to Professor Patrick Dessein, Cardiovascular Pathophysiology and Genomics Research Unit, School of Physiology, Faculty of Health Sciences, University of the Witwatersrand, Johannesburg, South Africa, PO Box 1012, Melville 2109, Johannesburg, South Africa; dessein@telkomsa.net
}

CVD risk factors. ${ }^{6}$ Congruent with this, although multiple risk factor equations are useful in determining the overall CVD risk among different populations, they often underestimate the actual risk in individual subjects. This is particularly evident in those who are at moderate risk according to major risk factor assessment equations. ${ }^{45}$ Consequently, based on reported evidence, two approaches that can refine CVD risk stratification are currently considered helpful in both European and American guidelines on CVD risk management. ${ }^{45}$ First, the use of biomarkers, particularly high-sensitivity $\mathrm{C}$ reactive protein concentrations, and second, cardiovascular imaging, including multidetector tomography coronary artery calcification scores (CACS) and carotid ultrasound; for the first time, carotid plaques are recognised to represent very high risk in the latest European guidelines on CVD prevention in clinical practice, ${ }^{4}$ where cardiovascular risk assessment is also not necessary because the patient is categorised into secondary prevention equivalent to patients with established CVD. Taking into account that patients with RA have two to three times more frequent asymptomatic carotid atherosclerosis compared with persons without RA, ${ }^{7-12}$ this will have a major impact on correct classification and hence treatment in patients with RA.

Would traditional risk factors as included in the mSCORE be expected to reliably reflect the actual CVD risk in RA? Recently reported evidence suggests that this may not be the case. One study showed that the mean Framingham score was as low as 7\% (low risk) in RA patients with carotid artery plaque despite it being associated with a 10-year incident CVD event rate of $\geq 39 \%$. $^{10}$ Indeed, Crowson and colleagues ${ }^{13}$ recently reported that the risk of CVD events is twofold and $65 \%$ higher than the Framingham score predicts in women and men with RA, respectively. The Reynolds risk score that additionally includes high-sensitivity C reactive protein concentrations and a family history of premature CVD revealed similar shortcomings. ${ }^{13}$ Using the area under the receiver operating characteristic curve in logistic regression analysis, traditional and non-traditional CVD risk factors associated similarly and additively with plaque prevalence. ${ }^{14}$ Solomon and colleagues $^{15}$ subsequently confirmed that the same applied when the risk of incident CVD events was examined. The associations of traditional risk factors with CVD event rates are weakened in RA. ${ }^{16}$ Indeed, the relation between lipids and CVD is at most inconsistent ${ }^{17}$ and in one report was in fact paradoxically inverse, in RA. ${ }^{18}$ A recent investigation disclosed that in contrast to their white counterparts, black Africans with RA experience no major conventional risk factor-atherosclerosis and systemic inflammation-atherosclerosis relations. ${ }^{19}$ This further argues against the reliance on risk factor profiles as currently recommended in evaluating actual CVD risk.

Treatment with methotrexate reduces the risk for CVD events by $\sim 20 \%$ in RA. $^{20}$ Indeed, systemic inflammation among patients with RA contributes substantially to increased CVD risk both through its adverse impact on traditional risk factors and direct effects on the vasculature. $^{21} 22$ Therefore, effective CVD risk management likely comprises not only adequate treatment of conventional risk factors but also tight disease activity control in RA.

With regard to the multiplier application as recommended by the EULAR task force, ${ }^{3}$ other recent reports revealed that the enhanced CVD risk is unlikely to be restricted to those patients with a disease duration of $>10$ years, neither to those who experience rheumatoid factor positivity and presumably also not to the small subgroup with extra-articular manifestations. ${ }^{23}$ The EULAR task force acknowledged that their approach was conservative. ${ }^{3}$ Taken together, evidence that was mostly reported after the EULAR recommendations for CVD risk management were published raises the possibility that their application could result in a substantial proportion of patients with RA at high risk for CVD remaining unidentified. ${ }^{23}$

However, are cardioprotective drugs effective in CVD risk management in RA? Indeed, there is a lack of hard evidence regarding CVD prevention in patients with RA and therefore many questions remain unanswered. In this regard, it is both noteworthy and promising that in a post hoc analysis of two prospective trials that assessed the impact of intensive compared with conventional treatment with statins on a CVD outcome, patients with and without inflammatory joint disease were found to experience comparable lipid-lowering effects and reduced CVD 
risk. ${ }^{24}$ Also, in a preventive cardio-rheuma clinic, two-thirds of patients referred for CVD risk evaluation required CVD prevention, ${ }^{11}$ which further reinforces the need for identifying patients at high risk. RA patients often sustain background marked systemic inflammation, altered lipid parameters and exposure to polypharmacy, all of which can influence optimal lipid-lowering treatment in CVD prevention. Despite the presence of these factors, treatment to lipid targets was successful in as many as $\sim 90 \%$ of patients with RA who required interventions with cardiovascular drugs. ${ }^{11}$ Experience and studies on clinical CVD prevention are warranted.

Corrales $e a^{25}$ compared the abilities of the mSCORE; carotid ultrasound determined advanced atherosclerosis and CACS in the identification of patients with RA who sustain high or very high CVD risk in the absence of established high or very high risk. Upon using the EULAR task force multiplier, the proportion of patients with high or very high risk increased by only $3.1 \%$, that is, from $11.6 \%$ to $14.7 \%$. High CACS $(>100)$ were observed in a mostly similar proportion of all patients, that is, $17.9 \%$, and in none with an mSCORE of $<1 \%$ (low risk). By contrast, $73.7 \%$ of all patients had ultrasonographically confirmed advanced atherosclerosis. Remarkably, this comorbidity was observed not only in $85 \%$ of patients at moderate risk (mSCORE $>1$ and $<5 \%$ ) but also in $33.3 \%$ of those with an mSCORE $<1 \%$. CACS and ultrasound findings correlated significantly. Nevertheless, even among the $41.2 \%$ with no detected coronary artery calcification, $57.5 \%$ had carotid plaques. Finally, in keeping with these findings, upon employing the presence of high/very high risk as determined by an mSCORE $>5$, a CACS of $>100$ or ultrasonographically determined advanced atherosclerosis as outcome variable, the sensitivities of an mSCORE $>5$, CACS $>100$ and ultrasonographically determined advanced atherosclerosis were $19.4 \%, 23.6 \%$ and $97.2 \%$, respectively. The findings on ultrasound in the Corrales study are largely similar to those recently reported by the same group in a larger cohort. ${ }^{26}$

Overall, in the Corrales study, by considering ultrasound findings in addition to the EULAR recommendations, the proportion of patients stratified as being at high or very high CVD risk increased from $22.1 \%$ to $77.9 \%$ or 3.5 -fold. Most importantly, in routine clinical settings these patients would generally not receive adequate preventive CVD risk treatment with the serious consequences this has.
Moreover, if up to a third of patients with RA without established high CVD risk and considered to be at low risk are actually at very high risk, it would appear at least reasonable to perform carotid ultrasound not only in those at intermediate risk but also in those with low risk according to the mSCORE. Although the respective proportion was only $13 \%$ in the previously reported Corrales study, ${ }^{26}$ even the absence of identifiable carotid artery plaque by ultrasound still does not fully exclude the possibility of prevalent significant coronary artery disease. ${ }^{27}$

Could carotid artery plaque associate with lower incident CVD event rates and why would CACS be less sensitive in discerning high risk in RA? Both carotid artery atherosclerosis and CACS predict incident CVD events beyond other risk factors in RA. ${ }^{28-30}$ Vulnerable plaques are more echolucent and typically have a lipid-rich core, macrophages and a low collagen content. ${ }^{31}$ By contrast, plaques that are less vulnerable to rupture are more echogenic and contain more collagen, dense fibrous tissue and various amounts of calcification and represent more advanced disease. ${ }^{31}$ Patients with RA experience a disease activity-related increased vulnerable plaque burden. ${ }^{12}$ It is therefore highly unlikely that reliance on the presence of carotid plaque translates into an overestimate of CVD risk in RA, and it is indeed expected that CACS are less sensitive in this context. The latter is, however, also recognised in non-RA subjects. $^{32}$

Upon considering the potential use of carotid ultrasonography in CVD risk stratification in RA, the following issues are equally relevant. In contrast to determining CACS, ultrasonography is inexpensive, does not require radiation and is considered cost-effective. ${ }^{33}$ Could the additional use of biomarkers ${ }^{34-37}$ be preferable to vascular imaging upon evaluating CVD risk in RA? Biomarkers of CVD risk are valuable in examining atherogenic mechanisms in $\mathrm{RA}^{34-36}$ but the identification of those that predict CVD events beyond other risk factors in this disease is in its early stages at present and requires intensive and careful exploration. ${ }^{37}$ Also, employing (presumably) a panel of useful biomarkers is likely to enhance the involved costs to a larger extent than performing carotid ultrasonography. Nevertheless, plaque represents advanced atherosclerosis and hence biomarkers that reflect enhanced atherogenesis in RA may well be needed to timely reclassify patients with RA in CVD risk groups and identify those at high risk at a stage prior to plaque occurrence. Finally, whereas plaque associates closely with coronary artery disease, increased carotid intimamedia thickness (CIMT) represents mostly high blood pressure-mediated arterial media hypertrophy and relates more strongly to left ventricular hypertrophy. ${ }^{31}$ Omission of CIMT results in the Corrales study would not be anticipated to alter the findings as only one of the patients with a CIMT $>0.9 \mathrm{~mm}$ did not have plaque.

The article by Corrales and colleagues does not allude to the limitations of their investigation that do, however, require further elucidation. Their cross-sectional design precludes drawing inferences on the direction of causality and, accordingly, the role of carotid ultrasonography in CVD risk assessment and management and reduction of cardiovascular event rates needs evaluation in a longitudinal study. Also, since the mean disease duration was 10.8 years it remains to be clarified whether carotid ultrasound is as helpful among patients with early disease versus those with long-standing disease in enhancing CVD risk stratification.

Improved risk stratification alone will not reduce cardiovascular event rates, unless it is accompanied by adequate CVD risk management in RA. Recently reported retrospective data suggest that the latter often does not occur ${ }^{38}$ and traditional risk factors are underdiagnosed and undertreated in $\mathrm{RA},{ }^{22}$ a situation that could in itself contribute to enhanced CVD risk. This deficiency is amply confirmed in a prospectively designed crosssectional investigation on CVD risk factor control in 836 patients with RA by Primdahl and colleagues, ${ }^{39}$ as also reported in Annals of Rheumatic Diseases. Most striking is that among the 644 patients without established CVD or diabetes, inadequate blood pressure and lipid control were documented in $35.8 \%$ and $55.4 \%$ of participants, respectively. Even more concerning is that among those with CVD and diabetes, these proportions were as high as $36.2 \%$ and $84.2 \%$ and $73.6 \%$ and $80.9 \%$, respectively. Clearly, effective and innovative measures aimed at improving systematic evaluation and treatment of unfavourable CVD risk factor profiles by healthcare providers in patients with RA are urgently needed. In line with the findings in the Corrales study, ${ }^{25}$ upon applying the EULAR task force multiplier, ${ }^{3}$ the proportion of patients with high or very high risk increased by only $3.6 \%$, that is, from $12.6 \%$ to $16.2 \%$.

Perhaps the most pertinent issue that requires clarification here is: who should 
take the responsibility to manage CVD risk in patients with RA? Enrolment in a preventive clinic with cardiologist involvement as currently done in a centre in Oslo, Norway, is likely to constitute one promising option in this regard. ${ }^{11}$ However, the establishment of such clinics may not be possible in less well-resourced countries. The Primdahl study investigators fully informed the patient, relevant hospital department and treating general practitioner (GP) about the participant's risk profile and subsequently referred the patient to their GP. In this regard, in The Netherlands, the GP now assesses and manages CVD risk in patients with RA, a process that is facilitated by embedding of recommendations in the corresponding relevant guideline. ${ }^{40}$ Again for patients who form part of less affluent societies, this may not be feasible as it involves extra direct and indirect costs. Should, at least in some settings, the treating rheumatologist manage CVD risk in addition to obtaining optimal disease activity control? ${ }^{41}$ At the very least, population or/and country-specific factors, including socioeconomic status, need to be accounted for in designing optimal and feasible CVD risk management strategies in RA.

In conclusion, whereas the mortality gap between patients with RA and the general population reportedly continues to widen, ${ }^{42}$ considering the findings in the Corrales and Primdahl studies could contribute to the implementation of potentially effective strategies in our attempts at reducing cardiovascular risk in RA.

\section{Competing interests None.}

Provenance and peer review Not commissioned; externally peer reviewed.

PHD and AGS contributed equally.

To cite Dessein PH, Semb AG. Ann Rheum Dis 2013; 72:1743-1746.

Received 1 June 2013

Revised 15 July 2013

Accepted 24 July 2013

Published Online First 9 August 2013

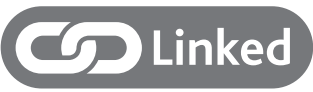

- http://dx.doi.org/10.1136/annrheumdis-2013203688

- http://dx.doi.org/10.1136/annrheumdis-2013203682

Ann Rheum Dis 2013;72:1743-1746. doi:10.1136/annrheumdis-2013-203911

\section{REFERENCES}

1 Avina-Zubieta JA, Choi HK, Sadatsafavi M, et al. Risk of cardiovascular mortality in patients with rheumatoid arthritis: a meta-analysis of observational studies. Arthritis Rheum 2008;59:1690-7.

2 Meune C, Touze E, Trinquart L, et al. High risk of clinical cardiovascular events in rheumatoid arthritis: levels of associations of myocardial infarction and stroke through a systematic review and meta-analysis. Arch Cardiovasc Dis 2010;103:253-61.

3 Peters MJL, Symmons DPM, McCarey, et al. EULAR evidence-based recommendations for cardiovascular risk management in patients with rheumatoid arthritis and other forms of inflammatory arthritis. Ann Rheum Dis 2010;69:325-31.

4 Perk J, De Backer G, Gohlke H, et al. European guidelines on cardiovascular disease prevention in clinical practice (version 2012). The Fifth Joint Task Force of the European Society of Cardiology and other Societies on Cardiovascular Disease Prevention in Clinical Practice (constituted by representatives of nine societies and by invited experts). Developed with the special contribution of the European Association for Cardiovascular Prevention \& Rehabilitation (EACPR). Atherosclerosis 2012;223:1-68.

5 Greenland P, Alpert JS, Beller GA, et al. 2010 ACC/ AHA guideline for assessment of cardiovascular risk in asymptomatic adults: a report of the American College of Cardiology Foundation/American Heart Association Task Force on Practice Guidelines. J Am Coll Cardiol 2010;56:e50-103.

6 Liao Y, McGee DL, Cooper RS, et al. How generalizable are coronary risk prediction models? Comparison of Framingham and two national cohorts. Am Heart J 1999;137:837-45.

7 Roman MJ, Moeller E, Davis A, et al. Preclinical carotid atherosclerosis in patients with rheumatoid arthritis. Ann Intern Med 2006;144:249-56.

8 Kobayashi H, Giles JT, Polak JT, et al. Increased prevalence of carotid artery atherosclerosis in rheumatoid arthritis is artery-specific. I Rheumatol 2010;37:730-9.

9 Aubry MC, Maradit-Kremers H, Reinalda MS, et al. Differences in atherosclerotic coronary heart disease between subjects with and without rheumatoid arthritis. J Rheumatol 2007;34:937-42.

10 Dessein $\mathrm{PH}$, Joffe BI, Veller MG, et al. Traditional and nontraditional cardiovascular risk factors are associated with atherosclerosis in rheumatoid arthritis. J Rheumatol 2005;32:435-42.

11 Rollefstad S, Kvien TK, Holme I, et al. Treatment to lipid targets in patients with inflammatory joint diseases in a preventive cardio-rheuma clinic. Ann Rheum Dis Published Online First: 22 December 2012. doi:10.1136/annrheumdis-2012-202789

12 Semb AG, Rollefstad S, Provan SA, et al. Carotid plaque characteristics and disease activity in rheumatoid arthritis. J Rheumatol 2013;40:359-68.

13 Crowson CS, Matteson EL, Roger VL, et al. Usefulness of risk scores to estimate the risk of cardiovascular disease in patients with rheumatoid arthritis. Am J Cardiol 2012;110:420-4.

14 Dessein PH, Norton GR, Woodiwiss AJ, et al. Influence of nonclassical cardiovascular risk factors on the accuracy of predicting subclinical atherosclerosis in rheumatoid arthritis. J Rheumatol 2007;34:943-51.

15 Solomon DH, Kremer J, Curtis JR, et al. Explaining the cardiovascular risk associated with rheumatoid arthritis: traditional risk factors versus markers of rheumatoid arthritis severity. Ann Rheum Dis 2012:69:1920-5

16 Gabriel SE. Heart disease and rheumatoid arthritis: understanding the risks. Ann Rheum Dis 2010;69 (Suppl 1):61-4

17 Semb AG, Kvien TK, Aastveit AH, et al. Lipids, myocardial infarction and ischaemic stroke in patients with rheumatoid arthritis in the Apolipoprotein-related Mortality Risk (AMORIS) Study. Ann Rheum Dis 2010:69:1996-2001.
18 Myasoedova E, Crowson CS, Kremers HM, et al. Lipid paradox in rheumatoid arthritis: the impact of serum lipid measures and systemic inflammation on the risk of cardiovascular disease. Ann Rheum Dis 2011;70:482-7.

19 Solomon A, Woodiwiss AJ, Abdool-Carrim AT, et al. The carotid artery atherosclerosis burden and its relation to cardiovascular risk factors in black and white Africans with established rheumatoid arthritis: a cross-sectional study. J Rheumatol 2012;39:1798-806.

20 Micha R, Imamura F, Wyler von Ballmoos $\mathrm{M}$, et al. Systematic review and meta-analysis of methotrexate use and risk of cardiovascular disease. Am J Cardiol 2011;52:1362-70.

21 Gabriel SE. Heart disease and rheumatoid arthritis: understanding the risks. Ann Rheum Dis 2010;69 (Suppl):i61-4.

22 Kitas GD, Gabriel SE. Cardiovascular disease in rheumatoid arthritis: state of the art and future perspectives. Ann Rheum Dis 2011;70:8-14.

23 Crowson CS, Gabriel SE. Towards improving cardiovascular risk management in patients with rheumatoid arthritis: the need for accurate risk assessment. Ann Rheum Dis 2011;70:

719-21

24 Semb AG, Kvien TK, DeMicco DA, et al. Effect of intensive lipid-lowering therapy on cardiovascula outcome in patients with and those without inflammatory joint disease. Arthritis Rheum 2012;64:2836-46.

25 Corrales A, Parra JA, Gonzalez-Juanatey C, et al. Cardiovascular risk stratification in rheumatic diseases: carotid ultrasound is more sensitive than Coronary Artery Calcification Score to detect subclinical atherosclerosis in patients with rheumatoid arthritis. Ann Rheum Dis 2013;72 1764-70.

26 Corrales A, Gonzalez-Juanatey C, Peiro ME, et al. Carotid ultrasound is useful for the cardiovascular risk stratification of patients with rheumatoid arthritis: results of a population-based study. Ann Rheum Dis Published Online First: 16 March 2013. doi:10.1136/annrheumdis-2012-203101

27 Brook RD, Bard RL, Patel S, et al. A negative carotid plaque area test is superior to other noninvasive atherosclerosis studies for reducing the likelihood of having underlying significant coronary artery disease. Arterioscler Thromb Vasc Biol 2006;26 656-62.

28 Gonzalez-Juanatey C, Llorca J, Martin J, et al. Carotid intima-media thickness predicts the development of cardiovascular events in patients with rheumatoid arthritis. Semin Arthritis Rheum 2009;38:366-71.

29 Evans MR, Escalante A, Battafarano DF, et al. Carotid atherosclerosis predicts incident acute coronary syndromes in rheumatoid arthritis. Arthritis Rheum 2011:63:1211-20.

30 Yiu KH, Mok MY, Wang S, et al. Prognostic role of coronary calcification in patients with rheumatoid arthritis and systemic lupus erythematosus. Clin Exp Rheumatol 2012:30:345-50.

31 Johnsen SH, Mathiesen EB. Carotid plaque compared with intima-media thickness as a predictor of coronary and cerebrovascular disease. Curr Cardiol Rep 2009;11:21-7.

32 Mancini GB, Dahlof B, Diez J. Surrogate markers for cardiovascular disease: structural markers. Circulation 2004;109(25 Suppl 1):IV22-30.

33 Makover ME, Ebrahim S. What is the best strategy for reducing deaths from heart disease? PLOS Medicine 2005;2:272-6.

34 Kozera L, Andrews J, Morgan AW. Cardiovascular risk and rheumatoid arthritis-the next step: differentiating true soluble markers of cardiovascular risk from surrogate measures of inflammation. Rheumatology 2011;50:1944-54. 
35 Rodriguez-Rodriguez L, Lopez-Mejias R, Garcia-Bermudez M, et al. Genetic markers of cardiovascular disease in rheumatoid arthritis. Mediators Inflamm 2012;2012: 574817.

36 Dessein PH, Norton GR, Woodiwiss AJ, et al. Independent relationship between circulating resistin concentrations and endothelial activation in rheumatoid arthritis. Ann Rheum Dis 2013;72:1586-8.

37 de Lemos JA, McGuire DK. Biomarkers in clinical trials. Can we move from fortune telling to disease profiling? Circulation 2011;124: 663-5.

38 Desai SS, Myles JD, Kaplan MJ. Suboptimal cardiovascular risk factor identification and management in patients with rheumatoid arthritis: a cohort analysis. Arthritis Res Ther 2012; 14:R270.

39 Primdahl J, Clausen J, Horslev-Petersen K. Results from systematic screening for cardiovascular risk in outpatients with rheumatoid arthritis in accordance with the EULAR recommendations. Ann Rheum Dis 2013:72:1771-6.
40 Banga JD, van Dijk JL, van Dis I, et al. NHG-standaard cardiovasculair risicomanagement (eerste herziening). Huisarts Wet 2012;55:14-28.

41 Provan SA, Semb AG, Hisdal J, et al. Remission is the goal for cardiovascular risk management in patients with rheumatoid arthritis: a cross-sectional comparative study. Ann Rheum Dis 2011;70: 812-7.

42 Gonzalez A, Maradit KH, Crowson CS, et al. The widening mortality gap between rheumatoid arthritis patients and the general population. Arthritis Rheum 2007:56:3583-7. 